\title{
Exploring the barriers to accessing care and treatment for HIV- infected children in India: A diagnostic study
}

\author{
Avina Sarna \\ Population Council \\ Jaleel Ahmad \\ Population Council \\ Glory Alexander \\ Vaishali Sharma Mahendra \\ Ashok Rau
}

See next page for additional authors

Follow this and additional works at: https://knowledgecommons.popcouncil.org/departments_sbsr-hiv

Part of the Demography, Population, and Ecology Commons, Family, Life Course, and Society Commons, International Public Health Commons, Maternal and Child Health Commons, and the Medicine and Health Commons

How does access to this work benefit you? Let us know!

\section{Recommended Citation}

Sarna, Avina, Jaleel Ahmad, Glory Alexander, Vaishali Sharma Mahendra, Ashok Rau, Arjun Singh, and Naomi Rutenberg. 2007. "Exploring the barriers to accessing care and treatment for HIV-infected children in India: A diagnostic study," Research update. New Delhi: Population Council. 


\section{Authors}

Avina Sarna, Jaleel Ahmad, Glory Alexander, Vaishali Sharma Mahendra, Ashok Rau, Arjun Singh, and Naomi Rutenberg 

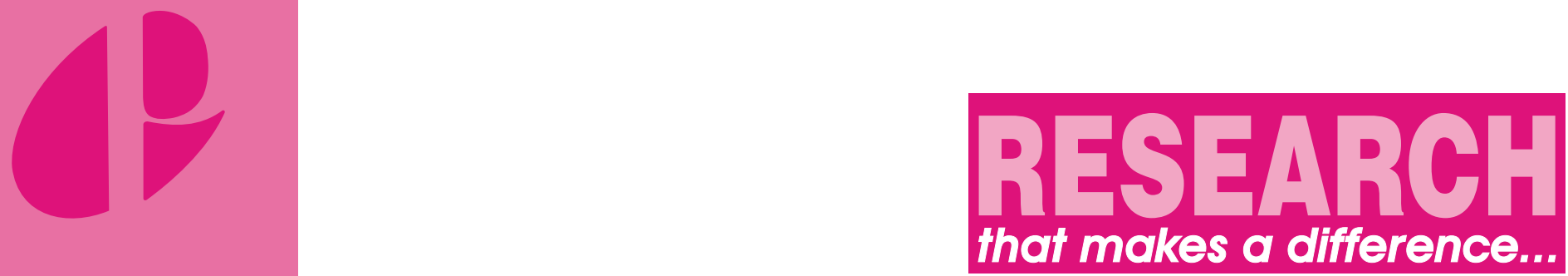

\section{Exploring the barriers to accessing care and treatment for HIV-infected children in India: a diagnostic study}

\section{Background}

Each year 27 million births take place in India. A conservative estimate of HIV prevalence of 1 percent among antenatal women translates to about 80,000 HIV-infected children being born annually. ${ }^{1}$ The latest figures from the National AIDS Control Organisation (NACO) show that there are about 5,600 documented HIV-positive children ( $0-14$ years) $)^{2}$ but a very small number are receiving antiretroviral therapy (ART). Researchers from the Population Council in collaboration with the Social Awareness Services Organisation (SASO), Asha Foundation and Freedom Foundation conducted an exploratory qualitative study in three high prevalence states of India: Manipur, Karnataka and Andhra Pradesh to (i) explore the facilitators and barriers to accessing care for HIV-positive children, and (ii) assess the current level of adherence to ART among children receiving treatment.

\section{Methods}

A cross-sectional study design was used. In-depth interviews were conducted with caregivers of HIV positive ( $<12$ years) and HIV positive adolescents (12 to 16 years) between June and December 2006 at selected treatment centres. A semi-structured questionnaire with open ended questions was used. Interviews were conducted in Kannada, Telegu and Manipuri.

For children less than 12 years of age, interviews were conducted with their primary caregivers. For those between 12 and 16 years, both the caregivers and adolescents were interviewed. Study participants were recruited from a convenience sample of community-based health facilities providing services for HIV positive persons and positive networks in the three states (Bangalore: 4; Bellary: 1; Kolara: 2; Udipi:1; Hyderabad: 4; Guntur:1 and Imphal:1).

This summary focuses on findings from caregivers of HIV-infected children less than 12 years of age.

\section{Characteristics of caregivers and children}

A total of 91 caregivers of children under 12 years of age who were taking ART (Karnataka: 44, Andhra Pradesh: 25, Manipur: 22) and 117 caregivers of children under 12 years of age not taking ART (Karnataka: 60, Andhra Pradesh: 33, Manipur: 24) were interviewed. The median age of caregivers was similar in both groups (31 years; IQR 28-38). The majority of caregivers interviewed were primary caregivers (ART: 97\%; Non-ART: 94\%), biological parents (ART: 74\%; Non-ART: $80 \%$ ) and female (ART: $75 \%$; Non-ART: $82 \%$ ). More than three-quarters of the caregivers were employed and more than 60 percent reported a household income of less than Rs 3000 per month in both groups. Manipur had the highest proportion of employed caregivers.

In both groups more than half the children being cared for by caregivers were boys (62\%). Among the children receiving ART ( $\mathrm{n}=91), 40$ percent were between 6 and 8 years of age and a further 51 percent of the children were 9 years old or more. Among those not receiving ART, a third each were less than 5 years old, between 6 and 8 years and over 9 years of age.

\section{Key Findings}

\begin{abstract}
Most HIV diagnosis among children takes place after children are 30 months or older

Less than a fifth of the children in the study had been diagnosed with HIV infection before 18 months of age (ART: 10\%; Non-ART: $16 \%$ ). The largest proportion had been tested between 30 and 60 months of age (ART: $40 \%$; Non-ART: $45 \%$ ); a fifth had been tested between 19-30 months and the remainder after 60 months (ART: $31 \%$; Non-ART: $25 \%)$.
\end{abstract}

The most frequently cited reason for seeking a HIV test for the child was the positive status of the parent(s) in both groups (ART: 56\%; Non-ART:

The Population Council, an international, nonprofit, nongovernmental organisation established in 1952, seeks to improve the well-being and reproductive health of current and future generations around the world and to help achieve a humane, equitable, and sustainable balance between people and resources. 
"I don't know what to do. It is not possible not to let him know. I don't know if I shall let him know when he can understand. If I tell him now, he may tell everyone that he had such and such and I don't want others to know of it."

Female caregiver, 39 years, Manipur

"After confirming husband's HIV status, I underwent an HIV test. The doctor asked to bring our child for diagnosis. He was also declared positive. This news spread in our village, people started to separate us. My neighbours didn't allow my child to play with their child, the village people didn't let us use the public borewell 5-10 times. I was not able to see this tragedy, I left my village" Female caregiver, 27 years, Bangalore

"I will give him medicines as long as I am there. If I am in good health then only I can provide him good care. If I die nobody will look after him."

Female caregiver, 25 years, Bangalore
$69 \%)$. Illness as a reason for seeking a HIV test was cited more often by caregivers of children receiving ART (ART: $37 \%$; Non-ART: $22 \%$ ).

HIV testing was conducted almost equally at public and private sector health facilities. In the public sector most testing took place at VCT centres (ART: $71 \%$ and Non ART: 63\%), while in the private sector most testing was done at private laboratories and clinics (ART: $48 \%$ and Non ART: $39 \%$ ). Less than a tenth of the tests were carried out at PPTCT sites in both groups.

Some caregivers face difficulties getting HIV diagnosis and referrals

More than a third of the caregivers reported facing problems in getting a confirmed HIV diagnosis and referral to treatment centres (ART: 34/91; Non-ART: 56/117). The most commonly cited problems faced were financial difficulties (ART: 28/34; Non-ART: 36/56), distance from health facilities (ART: 12/34; Non-ART: 9/56) and having to undertake multiple HIV tests at great cost (ART: 8/34; Non-ART:11/56).

\section{Disclosure of HIV status to children is low}

Less than a fifth of the children had been told of their HIV status (ART: 12/91; Non-ART: 14/117). Disclosure was highest among children between 9 to 12 years of age in both groups (ART: 11/12; NonART: 8/14). Reasons cited for not disclosing the HIV status to children were mostly fear of people's reaction, the child being too young to understand, and a fear that the child would tell others in the community. Most caregivers were unsure when they would disclose in the future. Internalized stigma was evident from responses; several caregivers feared discrimination by the family, in the community, teasing at school, child being thrown out of school, refusal of care at hospitals, loss of family support among others.

Around two-thirds of the caregivers in both groups reported that grandmothers, aunts and uncles within the family knew of the child's HIV status. Disclosure to siblings and cousins was lower and ranged between 14 to 24 percent in the two groups respectively. Less than a sixth had disclosed the child's status among friends and neighbors in the community.

\section{Stigma is still a barrier for some}

Caregivers were asked about stigmatizing experiences over the past 12 months.

Less than half the caregivers reported actually experiencing a stigmatizing experience (ART: $24 \%$; Non-ART: $41 \%$ ) in the family and community. The most commonly cited experiences were discrimination by the family, child not allowed to attend school, child not permitted to play with other children.
Less than six percent in both groups reported an experience of a health worker refusing to attend to the child (ART: $2 \%$; Non-ART: $4 \%$ ), waiting longer than other patients to be attended (ART: $6 \%$; NonART: $4 \%$ ), being tested without consent (ART: $6 \%$; Non-ART: $2 \%$ ) or disclosure of HIV status without consent (ART: 6\%;Non-ART: 0\%).

\section{Most families have more than one HIV infected person}

The majority of caregivers in both groups (ART: 93\%; Non ART: 96\%) reported other family members who were HIV positive. Two-thirds of the families had three or more HIV positive members (ART: 61\%; Non ART: 70\%). A higher proportion of families with children taking ART had family members taking ART (ART: 61\%; Non-ART: 44\%). More than four-fifths of family members in both groups were receiving ART at the same clinic as the children in their care (ART: $82.4 \%$; Non-ART: $84 \%$ ) with the same appointment dates.

Caregivers were asked if they knew of other HIVpositive children in their community. A little less than half of the caregivers knew of other infected children in their community (ART: $40 \%$; Non-ART: $43 \%)$.

\section{Caregiver knowledge of HIV transmission inadequate}

Just over a third of the caregivers could correctly identify all four routes of HIV transmission (ART: 37/91; Non-ART: 37/117). The most commonly cited routes were through blood and unclean equipment and mother-to-child transmission. A few caregivers mentioned mosquito bites, medications and eating food outside as possible routes of transmission.

Caregiver knowledge of ARV medications needs improvement; several caregivers not optimistic about continuing treatment life long

The majority of caregivers of children receiving $\operatorname{ART}(n=91)$ were aware that treatment needed to be taken life-long $(80 \%)$, however only 71 percent felt that their child would be able to continue treatment over the long term mostly because caregivers were unsure of their own survival, were unsure of the survival of the child, had financial insecurity and were unsure of the availability of free ARVs over the long term.

Although the caregivers interviewed in the study were the main persons responsible for supervising the child's ARV medications, only a quarter (24\%) could provide names of antiretroviral medications being given to the child; a further quarter $(26 \%)$ were able to describe the medications. Information on medications was obtained from physician's prescriptions in 35 percent of the interviews and medication wrappings in 13.5 percent. 
Overall, caregivers were aware of the consequences of treatment interruptions, e.g. occurrence of opportunistic infections (64/91), an increase in viral load (37/91), decrease in CD4 counts and immunity $(37 / 91)$ and the development of resistance to medications (26/91).

Caregivers in both groups expressed a need for more information on HIV/AIDS and treatment (ART: 89\%;Non-ART: 91\%).

\section{Caregivers' access antiretroviral medications} (ARV) equally from public and community-based clinics but turn to community-based NGOs for social support

Nearly half the children receiving ART ( $n=91)$ were accessing medications from public sector health facilities (48\%). An almost equal proportion (43\%) were accessing free medications from communitybased treatment centres. Around seven percent were receiving subsidized medications and less than two percent reported purchasing them from the market.

In both groups, the majority of caregivers reported receiving social support from outside the family (ART: 93\%; Non-ART: 82\%). Community-based NGOs were the most commonly cited source of support ( $>92$ percent) in both groups. Counselling, free medications and health checkups were the type of support most commonly reported.

\section{First line ARV treatment regimens used by the majority}

The vast majority of children on ART $(n=91)$ were receiving first line antiretroviral medications (94\%). Eighty-seven percent of the children were receiving a nevirapine-based treatment regimen with stavudine or zidovudine and lamivir, while six percent were getting an efavirenz-based regimen with stavudine or zidovudine and lamivir. The majority were using fixed dose combinations which require one dose in the morning and evening respectively, facilitating adherence to treatment. Children in Hyderabad had been on treatment for the shortest period of time as the program was just starting (median 6 months) compared to those in Bangalore (median 24 months) and Imphal (median 16 months).

\section{Overall adherence to treatment was high}

Adherence was assessed using the caregiver report. The majority of caregivers $(97 \%)$ reported a perfect adherence over the last two days for the children in their care $(n=91)$. Adherence was lower over longer periods of recall. Only 44 percent of caregivers reported never missing any doses of medications ever. Twenty-eight percent of children had missed a full day's medication at least once; 10 percent in the past one month. Fourteen percent reported a treatment interruption of more than a week's duration at least once.

The three most commonly cited reasons for missing any dose $(n=39)$ were the child being unwell $(8 / 39)$, the caregiver forgetting to give the child his/her medication (7/39) and being away from home (7/39). Reasons for longer treatment interruptions $(n=13)$ were mainly doctor's advice $(3 / 13)$, child having an infection $(2 / 13)$, caregiver/child being out of station $(2 / 13)$, child's refusal to take medications $(2 / 13)$ and financial problems (2/13).

Caregivers often face difficulties in administering medications and use incentives to get children to take medications

Nearly half the caregivers reported difficulties in administering medications to the children in their care $(36 / 91)$. The three most commonly cited problems were the child refusing to take medications $(11 / 36)$, breaking tablets to get the correct dose, as pediatric formulations are not available $(8 / 36)$, and children spitting out medications due to bad taste $(6 / 36)$.

Several caregivers reported using incentives $(\mathrm{n}=29)$ to get children to take medications, such as giving chocolates or biscuits $(15 / 29)$, and promising gifts $(8 / 29)$ or money $(5 / 29)$. A few caregivers $(n=25)$ reported having to use threats or force to get the child to take medications. Most commonly used threats were to reduce play time, giving the medication in an injection form, taking away chocolates etc. (15/25). A few reported beating the child $(4 / 25)$ and forcing medication into the mouth $(2 / 25)$.

\section{Cotrimoxazole prophylaxis used}

Three-quarters of the children not receiving ART (74\%) were taking preventive therapy (PT) with cotrimoxazole; the median duration was 12.5 months (IQR 4-27.5 months).

Although the majority accessed free PT from community-based NGO clinics (57\%) and public sector health facilities (11\%), nearly a third purchased them from chemists (29\%).

A third (32.6 percent) reported difficulties in giving children medications. As with ART, the most commonly cited reason was refusal by the child and spitting out.

\section{Health seeking behavior}

Almost three-fourths (73\%) of caregivers in both groups rated the general health of the HIV-infected children in their care as good or very good. Despite this assessment, nearly three-fourths of caregivers in both groups reported an episode of illness in the past 12 months that required medical attention (ART: 72\%; Non-ART:74\%). Nearly all sought medical care. Over a third of the caregivers in both
"Yes, he missed once when we went to the village; that was before last month. I took the medicines along with me but I did not give him because I did not want all others to see me giving medicine to avoid all their enquiries of why my child is taking medicine."

37 year old male caregiver, Hyderabad.

"....it is very difficult to break tablet, sir. There is no mark on any tablet. My child refuses to take also because of nausea, bad taste etc. I think there should be a medicine especially for children instead of this breaking." 29 year old female caregiver, Bangalore

"Yes, she had fever, sneezing, and coughs [illness in the past 12 months], for that we consulted the doctor and took medicine. Mostly, we will visit a local clinic for medical assistance because of the distance to this NGO clinic. Only for ARV treatment we will consult this NGO clinic"

23 year old female caregiver, Bangalore

\footnotetext{
"Yes, 2 to 3 times it happened [ missed clinic visits] so sir shouted at us, so from then I started borrowing from others."

Female caregiver, 29 years, Hyderabad
} 
groups visited a private health facility (ART: $38 \%$; Non-ART: $37 \%$ ), a third visited the communitybased NGO facility (ART: 34\%; Non-ART: 31\%) and a quarter visited public sector facilities (ART: $26 \%$; Non-ART: $26 \%$ ). The majority did not disclose the child's HIV status to health providers at clinics that were not their main treatment centres.

\section{Reaching health facilities is a problem formany}

Most caregivers used a bus for transportation to the community-based clinic (ART: 65\%; Non-ART: $71 \%$ ). For eighty percent of the caregivers it took less than 2 hours to get to the clinic. About twenty percent reported a commute of between 3-6 hours. More than three-quarters of the caregivers had to visit the clinic once a month. Twenty-four percent caregivers of children receiving ART and 38 percent of those not receiving ART reported having missed a clinic visit due to lack of money at least once. Several caregivers reported borrowing money to pay for transport.

\section{Programme implications and recommendations}

The exploratory study, conducted in three high prevalence states, provides important insights into access and treatment related issues concerning HIV infected children. The majority of children were tested later in life as a result of their parents' diagnosis of HIV infection, which is encouraging as families are being tested bringing children into care. However, an important gap emerges with regard to PPTCT as an entry point for diagnosis and access to treatment. Late testing also means delayed initiation of Cotrimoxazole prophylaxis which prevents life threatening opportunistic infections in infancy. The improved availability of PCR testing under the rapidly expanding national pediatric ART programme should facilitate early diagnosis of pediatric HIV infections ${ }^{3}$ and early initiation of preventive therapy; however health workers and NGOs would need to be more proactive. Testing in early childhood programmes such as immunization services, $\mathrm{MCH}$ clinics and well baby clinics needs to be explored.

Caregivers interviewed in this study were mostly primary caregivers and biological parents. The study reveals important gaps in caregiver knowledge with regard to medication names, routes of transmission and prevention of HIV transmission as also a certain lack of optimism. The majority of caregivers also expressed a need for more information on HIV infection and treatment. Improved preparation of caregivers through use of multiple sources of information such as counselling by health workers, parent to parent support groups, brochures and written materials and audio visual TV programmes at treatment centre waiting rooms could be evaluated. Strengthening counselling services for caregivers to facilitate disclosure of HIV status to children as they grow older, supporting caregivers to address fears of discrimination, and fostering optimism about their own survival and that of their children with treatment is needed.

The majority of families in the study had more than one HIV-positive member and most came from the lower economic status with household incomes of less than Rs 3000 per month, limiting resources available to access care. Barriers to accessing care included distance from health facilities and financial difficulties. Provision of treatment and care at the same clinic with the same appointments for family members facilitates access; a practice that can be used by health facilities as the programme scales up. Longer follow up periods (calling patients every 2 to 3 months) in stable patients as suggested by the NACO guidelines may be useful. ${ }^{4}$ Community based NGOs are an important source of social support for these families; these services need continued support and strengthening. Public sector treatment programmes may benefit from strengthening links with community based organizations. The lack of pediatric formulations needs to be addressed at a national programme level.

Although findings from convenience samples and small samples are not generalizable, this qualitative study provides important insights into the problems faced by caregivers of HIV-infected children and identifies important gaps in programmes. The ART program for children is expanding rapidly and studies with a larger number of children are recommended.

\section{(2) Population Council}

\section{FREEDOM FOUNDATION}
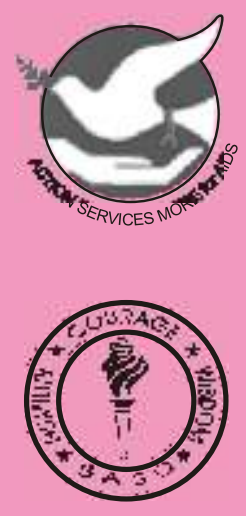

For further information please contact:

Dr Avina Sarna

Population Council

53, Lodi Estate

New Delhi - 110 003, India

Tel: 91-11-2469 9747/48

Fax: 91-11-2461 0912

Email: info-india@popcouncil.org

www.popcouncil.org

Contributors

Avina Sarna, Jaleel Ahmad,

Glory Alexander, Vaishali Mahendra,

Ashok Rau, Arjun Singh and

Naomi Rutenberg.

\section{References}

1. Proceedings of the NACO sponsored Working Group Meeting on Service Delivery, Delhi, 8 August, 2005.

2. NACO, Monthly Update on AIDS. www.nacoonline.org/facts_reportjuly.htm accessed on 24 March 2006.

3. D.T. Dunn, C.D. Brandt, A. Kirvine et al. The sensitivity of HIV-1 DNA polymerase chain reaction in the neonatal period and the relative contributions of intra-uterine and intrapartum transmission, AIDS, 1995, 9(9): F711.

4. Guidelines for HIV care and treatment in infants and children. NACO. November 2006

Suggested citation

Sarna, A., J. Ahmad, G. Alexander, V. Mahendra, A. Rau, A. Singh, and N. Rutenberg. 2007. “Exploring the barriers to accessing care and treatment for HIV-infected children: a diagnostic study", Research Update. Delhi. Population Council. 